1 Hacettepe Journal of Mathematics and Statistics

$\bigcap$ Volume 48 (1) (2019), 332-350

\title{
Moments and estimation of reduced Kies distribution based on progressive type-II right censored order statistics
}

\author{
Sanku Dey* ${ }^{*}$ Mazen Nassar ${ }^{\dagger}$ and Devendra Kumar ${ }^{\ddagger \S}$
}

\begin{abstract}
Based on progressive type-II censored samples, we first derive the recurrence relations for the single and product moments and then use these results to compute the means and variances of reduced Kies distribution (RKD), a new distribution, recently introduced by [21]. Next, we obtain the maximum likelihood estimators of the unknown parameter and the approximate confidence interval of the RKD. Finally, we consider Bayes estimation under the symmetric and asymmetric loss functions using gamma prior for the shape parameter. We have also derived two-sided Bayes probability interval (TBPI) and the highest posterior density (HPD) credible intervals of this distribution. Monte Carlo simulations are performed to compare the performances of the proposed methods, and a data set has been analyzed for illustrative purposes.
\end{abstract}

Keywords: Progressive type-II right censored order statistics, Single moments, Product moments, Recurrence relations, Reduced Kies distribution.

2000 AMS Classification: 62F15, 62F10, 62F99

Received : 10.09.2017 Accepted : 28.06.2018 Doi : 10.15672/HJMS.2018.611

*Department of Statistics, St. Anthony's College, Shillong-793001, Meghalaya, India, Email: sankud66@gmail.com

$\dagger$ Department of Statistics, Faculty of Commerce, Zagazig University, Egypt, Email: mezo10011@gmail.com

$\ddagger$ Department of Statistics, Central University of Haryana, Haryana, India, Email: devendrastats@gmail.com

$\S$ Corresponding Author. 


\section{Introduction}

Kies distribution was introduced by [19], in connection with the study of breaking strength of glass. Since then, a little work has taken place related to this distribution in different field of science and technology. An important characteristic of the Kies distribution is that its hazard function is decreasing, increasing and bathtub shaped where Weibull models are inappropriate. [21] considered a special case of the Kies distribution, which they termed as "'reduced Kies distribution"' and its probability density function (pdf) and cumulative density function $(\mathrm{CDF})$ are given by

$$
f(x ; \beta)=\beta x^{\beta-1}(1-x)^{-\beta-1} e^{-\left(\frac{x}{1-x}\right)^{\beta}}, \quad 0<x<1, \beta>0
$$

and

$$
F(x ; \beta)=1-e^{-\left(\frac{x}{1-x}\right)^{\beta}}, \quad 0<x<1, \beta>0 .
$$

This distribution can be viewed as a functional form of the Weibull distribution with shape parameter $\beta$ and it can be useful for modeling data sets with increasing and bathtub shaped hazard rate functions. Simple probability distributions generally do not exhibit bathtub-shaped failure rate, including Weibull, gamma, and log-normal. In most cases, bathtub shaped hazard functions have at least two parameters, whereas reduced Kies distribution has only one parameter which exhibit both increasing and bathtub shaped hazard rate. [21] observed that $\operatorname{RKD}(\beta)$ is a better model compared to the Weibull as well as its extended models such as beta Weibull distribution, beta generalised Weibull distribution etc. Interested readers may refer to [22] and [23] for an excellent exposure to the Kies distribution.

In case of complete data, it is necessary to continue the experiment until the last item/product failed. Very often, one may find that quite a number of items have very long lifetimes and the experiment continues for a very long period of time so much so that the results may no longer be of any interest or use. In such situations, it may be desirable to terminate the test prior to failure of all items under test. When test is discontinued prior to failure of all items, resulting observations will be called the censored sample. There exist various types of censored samples including Type-II, progressive Type-II, progressive first-failure censored samples and record values etc.

In this paper, we consider a more general censoring scheme called the progressive typeII right censoring scheme. Progressive type II right censoring is a useful scheme in which a specific fraction of individuals at risk may be removed from the experiment at each of several ordered failure times. There is a large body of literature dealing with progressive type II right censoring. For example, see [8], [10], [9] and [25] and the references therein. A Type-II progressively censored scheme can be expressed as: Suppose that $n$ units are put on life test at time 0 and the experimenter decides before hand the quantity $m$, the number of failures to be observed. Now at the time of first failure, $R_{1}$ units are randomly removed from the remaining $n-1$ surviving units. At the second failure, $R_{2}$ units from the remaining $n-2-R_{1}$ units are randomly removed. The test continues until the $m$-th failure. At this time, all remaining $R_{m}=n-m-R_{1}-R_{2}-\ldots-R_{m-1}$ units are removed. In this censoring scheme, $R_{i}$ and $m$ are previously fixed. The resulting $m$ ordered values, that are obtained as a consequence of this type of censoring are appropriately referred to as progressive Type-II censored ordered statistics. Note that, if $R_{1}=R_{2}=\ldots=$ $R_{m-1}=0$, so that $R_{m}=n-m$, this scheme reduces to conventional type II right censoring scheme. Also note that if $R_{1}=R_{2}=\ldots=R_{m}=0$, so that $m=n$, the progressively type II censoring scheme reduces to the case of no censoring (ordinary order statistics). 
Now in the view of (1.1) and (1.2), we have

$$
f(x)=\beta \sum_{p=0}^{\infty} \frac{(\beta+1)_{p} x^{\beta+p-1}}{p !}[1-F(x)],
$$

where $(e)_{k}=e(e+1) \cdots(e+k-1)$ denotes the ascending factorial. This equation will be exploited in order to derive some recurrence relations for the single and product moments of progressive Type-II right censored order statistics from the reduced Kies distribution. If the life times of an item are based on an absolutely continuous distribution function $F(x)$ with probability density function $f(x)$, the joint probability density function of the progressively censored failure times $X_{1: m: n}, X_{2: m: n}, \cdots, X_{m: m: n}$, is given by (see [8]).

$$
\begin{aligned}
& f_{X_{1: m: n}, X_{2: m: n}, \ldots, X_{m: m: n}}\left(x_{1}, x_{2}, \cdots, x_{m}\right) \\
= & A(n, m-1) \prod_{i=0}^{m} f\left(x_{i}\right)\left[1-F\left(x_{i}\right)\right]^{R_{i}} \\
& -\infty<x_{1}<x_{2}<\cdots<x_{m}<\infty
\end{aligned}
$$

where

$$
\begin{aligned}
& A(n, m-1) \\
= & n\left(n-R_{1}-1\right) \cdots\left(n-R_{1}-R_{2}-\cdots-R_{m-1}-m+1\right) .
\end{aligned}
$$

Let $X_{1}, X_{2}, \cdots, X_{n}$ be a random sample from the reduced kies distribution with pdf and cdf given in (1.1) and (1.2) respectively. The corresponding progressive type-II right censored order statistics with censoring scheme $\left(R_{1}, R_{2}, \cdots R_{m}\right), m \leq n$ will be

$$
X_{1: m: n}^{\left(R_{1}, R_{2}, \cdots, R_{m}\right)}, X_{2: m: n}^{\left(R_{1}, R_{2}, \cdots, R_{m}\right)}, \cdots, X_{m: m: n}^{\left(R_{1}, R_{2}, \cdots, R_{m}\right)} .
$$

The single moments of the progressive type-II right censored order statistics can be written as follows (see, [8]),

$$
\begin{aligned}
\mu_{i: m: n}^{\left(R_{1}, R_{2}, \cdots, R_{m}\right)^{(k)}} & =E\left[x_{i: m: n}^{\left.\left(R_{1}, R_{2}, \cdots, R_{m}\right)^{(k)}\right]}\right. \\
& =A(n, m-1) \iint \cdots \int_{0<x_{1}<x_{2}<\cdots<x_{m}<\infty} x_{i}^{k} f\left(x_{1}\right) \\
& \times\left[1-F\left(x_{1}\right]^{R_{1}} f\left(x_{2}\right)\left[1-F\left(x_{2}\right]^{R_{2}} f\left(x_{3}\right)\left[1-F\left(x_{3}\right]^{R_{3}} \cdots f\left(x_{m}\right)\right.\right.\right. \\
& \times\left[1-F\left(x_{m}\right]^{R_{m}} d x_{2} d x_{3} \cdots d x_{m},\right.
\end{aligned}
$$

where $f($.$) and F($.$) are given respectively in (1.1), (1.2), and A(n, m-1)$ as defined in (1.5). When $k=1$, the superscript in the notation of the mean of the progressive type-II right censored order statistics may be omitted without any confusion.

Recurrence relations for single and product moments for any continuous distribution can be used to compute all means and variances of a distribution. Many authors have obtained the recurrence relation for progressively type-II right censored order statistics for different distributions, see for example [15], [26], [29], [18], [3], [2], [30], [4], [1], [5], [6], [7], [16], [17] , [11], [28], [24], [12], [13], [14], [20] and the reference cited in.

The motivation of the paper is three fold: first, we derive recurrence relations for the single and product moments of the corresponding progressive Type-II right censored order statistics. These recurrence relations will allow one for the recursive computation of these moments for all sample sizes and all possible censoring schemes, second is to obtain the maximum likelihood estimators and confidence intervals of the unknown parameter of the model and third is to obtain the Bayes estimator under the symmetric and asymmetric loss functions using gamma prior for the shape parameter and two-sided Bayes 
probability interval (TBPI) and the highest posterior density (HPD) credible intervals. The uniqueness of this study comes from the fact that we provide explicit expressions for single and product moments using progressive type-II right censored order statistics along with parameter estimation using frequentist and Bayes.

The outline of this note is as follows: Recurrence relations for single moments of progressive type-II right censored order statistics from RKD are given in section 2. Further, section 3 describes the recurrence relations for product moments of progressive type-II right censored order statistics from RKD. The recurrence algorithm is carried out in section 4. In Section 5, we introduce the maximum likelihood estimation of the unknown parameter along with approximate confidence interval. In Section 6, We consider Bayesian estimation of the unknown parameter along with two-sided Bayes probability interval (TBPI) and the highest posterior density (HPD) credible intervals. A Monte Carlo simulation study is presented in Section 7 to evaluate the performances of the estimation method discussed in Sections 5 and 6. Then, in Section 8, we illustrate the methodology developed in this manuscript and the usefulness of the RKD based on progressive type-II right censored order statistics using a real data example. Finally, some concluding remarks are provided in Section 9.

\section{Recurrence relation for single moments}

In this section, we establish several new recurrence relations satisfied by the single moments of progressive type-II right censored order statistics from the reduced Kies distribution. These recurrence relations may be used to compute the means and variances of reduced Kies distribution based on progressive type-II right censored order statistics for all sample sizes $\mathrm{n}$ and all censoring schemes $\left(R_{1}, R_{2}, \cdots, R_{m}\right), m \leq n$.

2.1. Theorem. For $2 \leq m \leq n$ and $k \geq 0$,

$$
\begin{aligned}
\mu_{1: m: n}^{\left(R_{1}, R_{2}, \cdots, R_{m}\right)^{(k)}} & =\beta \sum_{p=0}^{\infty} \frac{(\beta+1)_{p}}{p !(k+\beta+p)}\left\{\left(n-R_{1}-1\right) \mu_{1: m-1: n}^{\left(R_{1}+1+R_{2}, \cdots, R_{m}\right)^{(k+\beta+p)}}\right. \\
& \left.+\left(1+R_{1}\right) \mu_{1: m: n}^{\left(R_{1}, R_{2}, \cdots, R_{m}\right)^{(k+\beta+p)}}\right\} .
\end{aligned}
$$

Proof. From equations (1.5) and (1.6), we have

$$
\begin{aligned}
\mu_{1: m: n}^{\left(R_{1}, R_{2}, \cdots, R_{m}\right)^{(k)}} & =A(n, m-1) \iint \cdots \int_{0<x_{1}<x_{2}<\cdots<x_{m}<\infty} \\
& \times L\left(x_{2}\right) f\left(x_{2}\right)\left[1-F\left(x_{2}\right]^{R_{2}} f\left(x_{3}\right)\left[1-F\left(x_{3}\right]^{R_{3}} \cdots f\left(x_{m}\right)\right.\right. \\
& \times\left[1-F\left(x_{m}\right]^{R_{m}} d x_{2} d x_{3} \cdots d x_{m},\right.
\end{aligned}
$$

where

$$
\begin{aligned}
L\left(x_{2}\right) & =\int_{0}^{x_{2}} x_{1}^{k} f\left(x_{1}\right)\left[1-F\left(x_{1}\right]^{R_{1}} d x_{1}\right. \\
& =\int_{0}^{x_{2}} x_{1}^{k}\left\{\beta \sum_{p=0}^{\infty} \frac{(\beta+1)_{p} x^{\beta+p-1}}{p !}\left[1-F\left(x_{1}\right]\right\}\left[1-F\left(x_{1}\right)\right]^{R_{1}} d x_{1}\right. \\
& =\beta \sum_{p=0}^{\infty} \frac{(\beta+1)_{p}}{p !} \int_{0}^{x_{2}} x_{1}^{k+\beta+p-1}\left[1-F\left(x_{1}\right)\right]^{R_{1}+1} d x_{1} .
\end{aligned}
$$


Integrating (2.3) by parts, we get after simplification

$$
\begin{aligned}
& =\beta \sum_{p=0}^{\infty} \frac{(\beta+1)_{p}}{p !(k+\beta+p)}\left\{\left[1-F\left(x_{2}\right)\right]^{R_{1}+1} x_{2}^{k+\beta+p}+\left(R_{1}+1\right) \int_{0}^{x_{2}} x_{1}^{k+\beta+p}\right. \\
& \left.\times \quad\left[1-F\left(x_{1}\right)\right]^{R_{1}} f\left(x_{1}\right) d x_{1}\right\} .
\end{aligned}
$$

Substituting the value of $L\left(x_{2}\right)$ from (2.4) in (2.2) and using (1.6), we have

$$
\begin{aligned}
\mu_{1: m: n}^{\left(R_{1}, R_{2}, \cdots, R_{m}\right)^{(k)}} & =\beta \sum_{p=0}^{\infty} \frac{(\beta+1)_{p}}{p !(k+\beta+p)}\left\{\iint \cdots \int x_{2}^{k+\beta+p}\left(1-F\left(x_{2}\right)\right)^{R_{1}+1} f\left(x_{2}\right)\right. \\
& \times\left(1-F\left(x_{2}\right)\right)^{R_{2}} \cdots f\left(x_{m}\right)\left(1-F\left(x_{m}\right)\right)^{R_{m}} \\
& \left.+\left(1+R_{1}\right) \mu_{1: m: n}^{\left(R_{1}, R_{2}, \cdots, R_{m}\right)^{(k+\beta+p)}}\right\} \\
& =\beta \sum_{p=0}^{\infty} \frac{(\beta+1)_{p}}{p !(k+\beta+p)}\left\{\left(n-R_{1}-1\right) \mu_{1: m-1: n}^{\left(R_{1}+1+R_{2}, \cdots, R_{m}\right)^{(k+\beta+p)}}\right. \\
& \left.+\left(1+R_{1}\right) \mu_{1: m: n}^{\left(R_{1}, R_{2}, \cdots, R_{m}\right)^{(k+\beta+p)}}\right\},
\end{aligned}
$$

rearranging the above equation gives the required result in (2.1).

2.2. Theorem. For $m=1, n=1,2, \cdots$ and $k \geq 0$,

$$
\mu_{1: 1: n}^{(n-1)^{(k)}}=n \beta \sum_{p=0}^{\infty} \frac{(\beta+1)_{p}}{p !(k+\beta+p)} \mu_{1: 1: n}^{(n-1)^{(k+\beta+p)}} .
$$

Proof. Similar to the proof of Theorem 2.1.

2.3. Remark. We may use the fact that the first progressive Type-II right censored order statistics is the same as the first usual order statistic from a sample of size $\mathrm{n}$, regardless of the censoring scheme employed.

2.4. Theorem. For $2 \leq i \leq m-1, m \leq n$ and $k \geq 0$,

$$
\begin{aligned}
\mu_{i: m: n}^{\left(R_{1}, R_{2}, \cdots, R_{m}\right)^{(k)}} & =\beta \sum_{p=0}^{\infty} \frac{(\beta+1)_{p}}{p !(k+\beta+p)}\left\{\left(n-R_{1}-R_{2}-\cdots-R_{i}-i\right)\right. \\
& \times \quad \mu_{i: m-1: n}^{\left(R_{1}, R_{2}, \cdots, R_{i-1}, R_{i}+R_{i+1}+1, R_{i+2}, \cdots, R_{m}\right)^{(k+\beta+p)}} \\
& +\left(1+R_{i}\right) \mu_{i: m: n}^{\left(R_{1}, R_{2}, \cdots, R_{m}\right)^{(k+\beta+p)}} \\
& -\left(n-R_{1}-R_{2}-\cdots-R_{i-1}-i+1\right) \\
\times & \left.\mu_{i-1: m-1: n}^{\left(R_{1}, R_{2}, \cdots, R_{i-2}, R_{i-1}+R_{i}+1, R_{i+1}, \cdots, R_{m}\right)^{(k+\beta+p)}}\right\} .
\end{aligned}
$$

Proof. Similar to the proof of Theorem 2.1.

2.5. Theorem. For $2 \leq m \leq n$, and $k \geq 0$,

$$
\begin{aligned}
\mu_{m: m: n}^{\left(R_{1}, R_{2}, \cdots, R_{m}\right)^{(k)}} & =\beta\left(1+R_{m}\right) \sum_{p=0}^{\infty} \frac{(\beta+1)_{p}}{p !(k+\beta+p)}\left\{\mu_{m: m: n}^{\left(R_{1}, R_{2}, \cdots, R_{m}\right)^{(k+\beta+p)}}\right. \\
& \left.-\mu_{m-1: m-1: n}^{\left(R_{1}, R_{2}, \cdots, R_{m-2}, R_{m-1}+R_{m}+1, R_{i+1}, \cdots, R_{m}\right)^{(k+\beta+p)}}\right\} .
\end{aligned}
$$

Proof. Similar to the proof of Theorem 2.1. 
2.6. Remark. Using these recurrence relations, we can obtain all the single moments of all progressive Type-II right censored order statistics for all sample sizes and censoring schemes $\left(R_{1}, R_{2}, \cdots, R_{m}\right)$ in a sample recursive manner.

Deductions: For the special case $R_{1}=R_{2}=\cdots=R_{m}=0$ so that $m=n$ in which the progressive censored order statistics become the usual order statistics $X_{1: n}, X_{2: n}, \cdots$, $X_{n: n}$, then

(i) From Eq. (2.1): For $k \geq 0$,we get

$$
\mu_{1: n}^{(k)}=\beta \sum_{p=0}^{\infty} \frac{(\beta+1)_{p}}{p !(k+\beta+p)}\left\{\mu_{1: n}^{k}+(n-1) \mu_{1: n-1: n}^{(1,0,0, \cdots, 0)^{(k+\beta+p)}}\right\} .
$$

(ii) From Eq. (2.6): For $k \geq 0$, we get

$$
\begin{aligned}
\mu_{i: n}^{(k)} & =\beta \sum_{p=0}^{\infty} \frac{(\beta+1)_{p}}{p !(k+\beta+p)}\left\{\mu_{i: n}^{(k+\beta+p)}+(n-i) \mu_{i: n}^{(k+\beta+p)}\right. \\
& \left.-(n-i+1) \mu_{i-1: n}^{(k+\beta+p)}\right\}
\end{aligned}
$$

\section{Recurrence relation for product moments}

In this section, we establish some recurrence relations for product moments of the progressive type-II right censored order statistics from the reduced Kies distribution. The $(i, j)^{t h}$ product moment of the progressive type-II right censored order statistics can be written as

$$
\begin{aligned}
\mu_{i, j: m: n}^{\left(R_{1}, R_{2}, \cdots, R_{m}\right)} & =E\left[x_{i: m: n}^{\left(R_{1}, R_{2}, \cdots, R_{m}\right)} x_{j: m: n}^{\left(R_{1}, R_{2}, \cdots, R_{m}\right)}\right] \\
& =A(n, m-1) \iint \cdots \int_{0<x_{1}<x_{2}<\cdots<x_{m}<\infty} x_{i} x_{j} f\left(x_{1}\right)\left[1-F\left(x_{1}\right)\right]^{R_{1}} \\
& \times f\left(x_{2}\right)\left[1-F\left(x_{2}\right)\right]^{R_{2}} \cdots f\left(x_{m}\right)\left[1-F\left(x_{m}\right)\right]^{R_{m}} d x_{1} d x_{2} d x_{3} \cdots d x_{m},
\end{aligned}
$$

where $f($.$) and F($.$) are given respectively in (1.1) and (1.2) and A(n, m-1)$ is defined in (1.5).

3.1. Theorem. For $1 \leq i<j \leq m-1$ and $m \leq n$,

$$
\begin{aligned}
& \mu_{i: m: n}^{\left(R_{1}, R_{2}, \cdots, R_{m}\right)}= \beta\left(R_{j}+1\right) \sum_{p=0}^{\infty} \frac{(\beta+1)_{p}}{p !(\beta+p)}\left\{\mu_{i, j: m: n}^{\left(R_{1}, R_{2}, \cdots, R_{m}\right)^{(1, \beta+p)}}\right. \\
&+\left(n-R_{1}-1-\cdots-R_{j}-j\right) \\
& \times \mu_{i, j: m-1: n}^{\left(R_{1}, R_{2}, \cdots, R_{j-1}, R_{j}+R_{j+1}+1, \cdots R_{m}\right)^{(1, \beta+p)}} \\
&-\left(n-R_{1}-1-\cdots-R_{j-1}-j+1\right) \\
&\left.\times \quad \mu_{i, j-1: m-1: n}^{\left(R_{1}, R_{2}, \cdots, R_{j-1}+R_{j}+1, \cdots, R_{m}\right)^{(1, \beta+p)}}\right\}
\end{aligned}
$$

Proof. Using (1.3) and (1.6), we have

$$
\begin{aligned}
\mu_{i: m: n}^{\left(R_{1}, R_{2}, \cdots, R_{m}\right)} & =A(n, m-1) \iint \cdots \int_{0<x_{1}<\cdots<x_{j-1}<x_{j+1}<\cdots<x_{m}<\infty} \\
& \times\left\{\int_{x_{j-1}}^{x_{j+1}} \beta \sum_{p=0}^{\infty} \frac{(\beta+1)_{p}}{p !} x_{j}^{\beta+p-1}\left[1-F\left(x_{j}\right)\right]^{R_{j}+1} d x_{j}\right\} x_{i} f\left(x_{1}\right) \\
& \times\left[1-F\left(x_{1}\right]^{R_{1}} \cdots f\left(x_{j-1}\right)\left[1-F\left(x_{j-1}\right]^{R_{j-1}} f\left(x_{j+1}\right)\left[1-F\left(x_{j+1}\right]^{R_{j+1}}\right.\right.\right. \\
(3.3) & \times \cdots f\left(x_{m}\right)\left[1-F\left(x_{m}\right]^{R_{m}} d x_{1} d x_{2} \cdots d x_{j-1} d x_{j+1} \cdots d x_{m} .\right.
\end{aligned}
$$


Integrating the innermost integral by parts, we obtain

$$
\begin{aligned}
& \beta \sum_{p=0}^{\infty} \frac{(\beta+1)_{p}}{p !} \int_{x_{j-1}}^{x_{j+1}} x^{\beta+p-1}\left[1-F\left(x_{j}\right)\right]^{R_{j}+1} d x_{j} \\
& =\beta \sum_{p=0}^{\infty} \frac{(\beta+1)_{p}}{p !(\beta+p)}\left\{x_{j+1}^{\beta+p}\left[1-F\left(x_{j+1}\right)\right]^{1+R_{j}}\right. \\
& -x_{j-1}^{\beta+p}\left[1-F\left(x_{j-1}\right)\right]^{1+R_{j}}+\left(1+R_{j}\right) \\
& \left.\times \int_{x_{j-1}}^{x_{j+1}}\left[1-F\left(x_{j}\right)\right]^{R_{j}} f\left(x_{j}\right) x_{j}^{\beta+p} d x_{j}\right\},
\end{aligned}
$$

which, when substituted into equation (3.3) and using (3.1), we have

$$
\begin{aligned}
\mu_{i: m: n}^{\left(R_{1}, R_{2}, \cdots, R_{m}\right)} & =\beta \sum_{p=0}^{\infty} \frac{(\beta+1)_{p}}{p !(\beta+p)}\left\{\left(n-R_{1}-1-\cdots-R_{j}-j\right)\right. \\
& \times \quad \mu_{i, j: m-1: n}^{\left(R_{1}, R_{2}, \cdots, R_{j-1}, R_{j}+R_{j+1}+1, \cdots R_{m}\right)^{(1, \beta+p)}} \\
& -\left(n-R_{1}-1-\cdots-R_{j-1}-j+1\right) \\
& \times \mu_{i, j-1: m-1: n}^{\left(R_{1}, R_{2}, \cdots, R_{j-1}+R_{j}+1, \cdots, R_{m}\right)^{(1, \beta+p)}} \\
& \left.+\left(R_{j}+1\right) \mu_{i, j: m: n}^{\left(R_{1}, R_{2}, \cdots, R_{m}\right)^{(1, \beta+p)}}\right\} .
\end{aligned}
$$

Upon rearrangement of this equation, we obtain the relation in (3.2).

3.2. Theorem. For $1 \leq i \leq m-1$ and $m \leq n$,

$$
\begin{aligned}
\mu_{i: m: n}^{\left(R_{1}, R_{2}, \cdots, R_{m}\right)}= & \beta \sum_{p=0}^{\infty} \frac{(\beta+1)_{p}}{p !(\beta+p)}\left\{\left(R_{m}+1\right) \mu_{i, m: m: n}^{\left(R_{1}, R_{2}, \cdots, R_{m}\right)^{(1, \beta+p)}}\right. \\
& \left(n-R_{1}-1-\cdots-R_{m-1}-m+1\right) \\
\times \quad & \left.\mu_{i, m-1: m-1: n}^{\left(R_{1}, R_{2}, \cdots, R_{m-1}+R_{m}+1, \cdots, R_{m}\right)^{(1, k+p)}}\right\} .
\end{aligned}
$$

Proof. Similar to the proof of Theorem 3.1.

3.3. Remark. Using these recurrence relations, we can obtain all the product moments of progressive type-II right censored order statistics for all sample sizes and censoring schemes $\left(R_{1}, R_{1}, \cdots, R_{m}\right)$.

\section{Recursive algorithm}

Using the recurrence relations established in Sections 2 and 3, the means and variances of all progressive type-II right censored order statistics from the reduced Kies distribution can be readily computed as follows:

All the first and second order moments with $m=1$ for all sample sizes $n$ can be obtained by setting $k=0$ in equation (2.5) and then again setting $k=1$ in the same equation. Next using equation (2.1), we can determine all the moments of the form $\mu_{1,2: n}^{\left(R_{1}, R_{2}\right)}, n=2,3 \cdots$, which can in turn be used again with equation (2.1), to determine all moments of the form $\mu_{1,2: n}^{\left(R_{1}, R_{2}\right)^{2}}, n=2,3 \cdots$. Equation (2.7) can then be used to obtain $\mu_{2,2: n}^{\left(R_{1}, R_{2}\right)}$ for all $R_{1}, R_{2}$ and $n \geq 2$ and these values can be used to obtain all moments of the form $\mu_{1,2: n}^{\left(R_{1}, R_{2}\right)^{2}}$ by using equation (2.7) again. Equation (2.1) can now be used again to obtain $\mu_{1,3: n}^{\left(R_{1}, R_{2}, R_{3}\right)}, \mu_{1,3: n}^{\left(R_{1}, R_{2}, R_{3}\right)^{2}}$ for all $n, R_{1}, R_{2}$ and $R_{3}$ and equation (2.7)can be used next to obtain all moments of the form $\mu_{2,3: n}^{\left(R_{1}, R_{2}, R_{3}\right)}, \mu_{2,3: n}^{\left(R_{1}, R_{2}, R_{3}\right)^{2}}$. Finally, equation (2.7) can be 
used to obtain all moments of the form $\mu_{3,3: n}^{\left(R_{1}, R_{2}, R_{3}\right)}, \mu_{3,3: n}^{\left(R_{1}, R_{2}, R_{3}\right)^{2}}$.

This process can be continued until all desired first and second order moments and hence all variances are obtained.

Table 1. Means of progressively Type-II right censored order statistics.

\begin{tabular}{|c|c|c|c|c|c|c|c|}
\hline \multicolumn{8}{|c|}{$\beta=2$} \\
\hline$m \downarrow$ & $n \downarrow$ & Scheme & & & Mean & & \\
\hline 5 & 2 & $(0,3)$ & 0.041723 & 0.072175 & & & \\
\hline 5 & 2 & $(3,0)$ & 0.041723 & 0.250621 & & & \\
\hline 8 & 2 & $(6,0)$ & 0.021305 & 0.196329 & & & \\
\hline 8 & 2 & $(0,6)$ & 0.021305 & 0.032634 & & & \\
\hline 10 & 2 & $(8,0)$ & 0.018253 & 0.172537 & & & \\
\hline 10 & 2 & $(0,8)$ & 0.018253 & 0.047385 & & & \\
\hline 12 & 2 & $(10,0)$ & 0.013252 & 0.177032 & & & \\
\hline 12 & 2 & $(0,10)$ & 0.013252 & 0.039572 & & & \\
\hline 15 & 2 & $(13,0)$ & 0.021425 & 0.172439 & & & \\
\hline 15 & 2 & $(0,13)$ & 0.021425 & 0.036125 & & & \\
\hline 18 & 2 & $(16,0)$ & 0.016234 & 0.169932 & & & \\
\hline 18 & 2 & $(0,16)$ & 0.016234 & 0.029620 & & & \\
\hline 20 & 2 & $(18,0)$ & 0.011772 & 0.175931 & & & \\
\hline 20 & 2 & $(0,18)$ & 0.011772 & 0.023972 & & & \\
\hline 5 & 3 & $(2,0,0)$ & 0.041720 & 0.113935 & 0.278143 & & \\
\hline 5 & 3 & $(0,0,2)$ & 0.041720 & 0.075434 & 0.276428 & & \\
\hline 8 & 3 & $(5,0,0)$ & 0.029243 & 0.096352 & 0.306950 & & \\
\hline 8 & 3 & $(0,0,5)$ & 0.029243 & 0.040270 & 0.071275 & & \\
\hline 10 & 3 & $(7,0,0)$ & 0.016306 & 0.099054 & 0.343772 & & \\
\hline 10 & 3 & $(0,0,7)$ & 0.016306 & 0.041004 & 0.062801 & & \\
\hline 12 & 3 & $(9,0,0)$ & 0.023762 & 0.091843 & 0.254153 & & \\
\hline 12 & 3 & $(0,0,9)$ & 0.023762 & 0.034956 & 0.063072 & & \\
\hline 15 & 3 & $(12,0,0)$ & 0.021934 & 0.088032 & 0.262183 & & \\
\hline 15 & 3 & $(0,0,12)$ & 0.021934 & 0.031835 & 0.041152 & & \\
\hline 18 & 3 & $(15,0,0)$ & 0.012201 & 0.091148 & 0.260841 & & \\
\hline 18 & 3 & $(0,0,15)$ & 0.012201 & 0.028103 & 0.036110 & & \\
\hline 20 & 3 & $(17,0,0)$ & 0.009083 & 0.090581 & 0.249481 & & \\
\hline 20 & 3 & $(0,0,17)$ & 0.009083 & 0.024065 & 0.038214 & & \\
\hline 5 & 4 & $(1,0,0,0)$ & 0.041042 & 0.090662 & 0.168825 & 0.410550 & \\
\hline 5 & 4 & $(0,0,0,1)$ & 0.041042 & 0.071167 & 0.124023 & 0.201833 & \\
\hline 8 & 4 & $(4,0,0,0)$ & 0.023810 & 0.073911 & 0.205070 & 0.301149 & \\
\hline 8 & 4 & $(0,0,0,4)$ & 0.023810 & 0.052061 & 0.074052 & 0.099701 & \\
\hline 10 & 4 & $(6,0,0,0)$ & 0.016113 & 0.071152 & 0.140319 & 0.290661 & \\
\hline 10 & 4 & $(0,0,0,6)$ & 0.016113 & 0.043860 & 0.061734 & 0.081195 & \\
\hline 12 & 4 & $(8,0,0,0)$ & 0.013762 & 0.073054 & 0.142933 & 0.294821 & \\
\hline 12 & 4 & $(0,0,0,8)$ & 0.013762 & 0.039928 & 0.053028 & 0.063427 & \\
\hline 15 & 4 & $(11,0,0,0)$ & 0.031830 & 0.073663 & 0.147430 & 0.290271 & \\
\hline 15 & 4 & $(0,0,0,11)$ & 0.031830 & 0.031846 & 0.042835 & 0.050728 & \\
\hline 18 & 4 & $(14,0,0,0)$ & 0.015221 & 0.062283 & 0.145327 & 0.311052 & \\
\hline 18 & 4 & $(0,0,0,14)$ & 0.015221 & 0.024201 & 0.034028 & 0.040475 & \\
\hline 20 & 4 & $(16,0,0,0)$ & 0.009117 & 0.057631 & 0.132802 & 0.283144 & \\
\hline 20 & 4 & $(0,0,0,16)$ & 0.009117 & 0.024240 & 0.030329 & 0.047301 & \\
\hline 5 & 5 & $(0,0,0,0,0)$ & 0.051683 & 0.070176 & 0.129787 & 0.211832 & 0.403193 \\
\hline 8 & 5 & $(3,0,0,0,0)$ & 0.027094 & 0.063837 & 0.199263 & 0.199330 & 0.398422 \\
\hline 8 & 5 & $(0,0,0,0,3)$ & 0.027094 & 0.051054 & 0.074056 & 0.104373 & 0.158304 \\
\hline 10 & 5 & $(5,0,0,0,0)$ & 0.017884 & 0.061539 & 0.154028 & 0.197502 & 0.379529 \\
\hline 10 & 5 & $(0,0,0,0,5)$ & 0.017884 & 0.043805 & 0.063183 & 0.081104 & 0.103304 \\
\hline 12 & 5 & $(7,0,0,0,0)$ & 0.020523 & 0.063056 & 0.212178 & 0.193653 & 0.472983 \\
\hline 12 & 5 & $(0,0,0,0,7)$ & 0.020523 & 0.038402 & 0.050117 & 0.063062 & 0.084025 \\
\hline 15 & 5 & $(10,0,0,0,0)$ & 0.020311 & 0.057324 & 0.103851 & 0.190437 & 0.411947 \\
\hline 15 & 5 & $(0,0,0,0,10)$ & 0.020311 & 0.039542 & 0.046210 & 0.051183 & 0.063826 \\
\hline 18 & 5 & $(13,0,0,0,0)$ & 0.009104 & 0.050284 & 0.108204 & 0.180852 & 0.415738 \\
\hline 18 & 5 & $(0,0,0,0,13)$ & 0.009104 & 0.021095 & 0.038431 & 0.049627 & 0.050642 \\
\hline 20 & 5 & $(15,0,0,0,0)$ & 0.008630 & 0.056418 & 0.108432 & 0.186549 & 0.485429 \\
\hline 20 & 5 & $(0,0,0,0,15)$ & 0.008630 & 0.039843 & 0.038754 & 0.047652 & 0.087434 \\
\hline
\end{tabular}


Table 2. Means of progressively Type-II right censored order statistics.

\begin{tabular}{|c|c|c|c|c|c|c|c|}
\hline \multicolumn{8}{|c|}{$\beta=4$} \\
\hline$m \downarrow$ & $n \downarrow$ & Scheme & & & Mean & & \\
\hline 5 & 2 & $(0,3)$ & 0.052113 & 0.213631 & & & \\
\hline 5 & 2 & $(3,0)$ & 0.052113 & 0.310047 & & & \\
\hline 8 & 2 & $(6,0)$ & 0.030174 & 0.301174 & & & \\
\hline 8 & 2 & $(0,6)$ & 0.030174 & 0.069876 & & & \\
\hline 10 & 2 & $(8,0)$ & 0.031010 & 0.301632 & & & \\
\hline 10 & 2 & $(0,8)$ & 0.031010 & 0.051042 & & & \\
\hline 12 & 2 & $(10,0)$ & 0.021273 & 0.314250 & & & \\
\hline 12 & 2 & $(0,10)$ & 0.021273 & 0.051634 & & & \\
\hline 15 & 2 & $(13,0)$ & 0.021225 & 0.302284 & & & \\
\hline 15 & 2 & $(0,13)$ & 0.021225 & 0.041229 & & & \\
\hline 18 & 2 & $(16,0)$ & 0.020318 & 0.280847 & & & \\
\hline 18 & 2 & $(0,16)$ & 0.020318 & 0.031054 & & & \\
\hline 20 & 2 & $(18,0)$ & 0.019134 & 0.341186 & & & \\
\hline 20 & 2 & $(0,18)$ & 0.019134 & 0.032832 & & & \\
\hline 5 & 3 & $(2,0,0)$ & 0.049102 & 0.210431 & 0.401152 & & \\
\hline 5 & 3 & $(0,0,2)$ & 0.049102 & 0.201043 & 0.210116 & & \\
\hline 8 & 3 & $(5,0,0)$ & 0.031028 & 0.191050 & 0.401432 & & \\
\hline 8 & 3 & $(0,0,5)$ & 0.031028 & 0.069032 & 0.190320 & & \\
\hline 10 & 3 & $(7,0,0)$ & 0.029875 & 0.178320 & 0.3956375 & & \\
\hline 10 & 3 & $(0,0,7)$ & 0.029875 & 0.051023 & 0.0988527 & & \\
\hline 12 & 3 & $(9,0,0)$ & 0.021029 & 0.149025 & 0.3783066 & & \\
\hline 12 & 3 & $(0,0,9)$ & 0.021029 & 0.054193 & 0.0710345 & & \\
\hline 15 & 3 & $(12,0,0)$ & 0.017843 & 0.139852 & 0.3698548 & & \\
\hline 15 & 3 & $(0,0,12)$ & 0.017843 & 0.039985 & 0.0501142 & & \\
\hline 18 & 3 & $(15,0,0)$ & 0.014325 & 0.129984 & 0.360012 & & \\
\hline 18 & 3 & $(0,0,15)$ & 0.014325 & 0.029959 & 0.042073 & & \\
\hline 20 & 3 & $(17,0,0)$ & 0.013062 & 0.125080 & 0.345211 & & \\
\hline 20 & 3 & $(0,0,17)$ & 0.013062 & 0.024643 & 0.039853 & & \\
\hline 5 & 4 & $(1,0,0,0)$ & 0.048104 & 0.132273 & 0.241104 & 0.498623 & \\
\hline 5 & 4 & $(0,0,0,1)$ & 0.048104 & 0.119984 & 0.198953 & 0.311032 & \\
\hline 8 & 4 & $(4,0,0,0)$ & 0.030218 & 0.112260 & 0.238421 & 0.468432 & \\
\hline 8 & 4 & $(0,0,0,4)$ & 0.030218 & 0.069852 & 0.140542 & 0.150436 & \\
\hline 10 & 4 & $(6,0,0,0)$ & 0.029844 & 0.108873 & 0.22062 & 0.450417 & \\
\hline 10 & 4 & $(0,0,0,6)$ & 0.029844 & 0.058426 & 0.080113 & 0.128421 & \\
\hline 12 & 4 & $(8,0,0,0)$ & 0.020115 & 0.097431 & 0.226542 & 0.449853 & \\
\hline 12 & 4 & $(0,0,0,8)$ & 0.020115 & 0.041854 & 0.072104 & 0.090438 & \\
\hline 15 & 4 & $(11,0,0,0)$ & 0.016420 & 0.093852 & 0.215490 & 0.440462 & \\
\hline 15 & 4 & $(0,0,0,11)$ & 0.016420 & 0.032052 & 0.050842 & 0.070114 & \\
\hline 18 & 4 & $(14,0,0,0)$ & 0.013054 & 0.090114 & 0.210113 & 0.439912 & \\
\hline 18 & 4 & $(0,0,0,14)$ & 0.013054 & 0.027043 & 0.041032 & 0.057083 & \\
\hline 20 & 4 & $(16,0,0,0)$ & 0.012056 & 0.089215 & 0.205890 & 0.436782 & \\
\hline 20 & 4 & $(0,0,0,16)$ & 0.012056 & 0.024054 & 0.037852 & 0.051201 & \\
\hline 5 & 5 & $(0,0,0,0,0)$ & 0.047134 & 0.110895 & 0.191013 & 0.310532 & 0.531052 \\
\hline 8 & 5 & $(3,0,0,0,0)$ & 0.029042 & 0.087552 & 0.172728 & 0.283820 & 0.521684 \\
\hline 8 & 5 & $(0,0,0,0,3)$ & 0.029042 & 0.062845 & 0.110405 & 0.145656 & 0.213482 \\
\hline 10 & 5 & $(5,0,0,0,0)$ & 0.024020 & 0.081095 & 0.167046 & 0.282608 & 0.514021 \\
\hline 10 & 5 & $(0,0,0,0,5)$ & 0.024020 & 0.049720 & 0.078632 & 0.120676 & 0.158226 \\
\hline 12 & 5 & $(7,0,0,0,0)$ & 0.020132 & 0.078010 & 0.163101 & 0.278743 & 0.510045 \\
\hline 12 & 5 & $(0,0,0,0,7)$ & 0.020132 & 0.041201 & 0.064322 & 0.090221 & 0.127035 \\
\hline 15 & 5 & $(10,0,0,0,0)$ & 0.016310 & 0.074135 & 0.160236 & 0.274888 & 0.506021 \\
\hline 15 & 5 & $(0,0,0,0,10)$ & 0.016310 & 0.032830 & 0.050623 & 0.070108 & 0.091026 \\
\hline 18 & 5 & $(13,0,0,0,0)$ & 0.013741 & 0.071565 & 0.156662 & 0.264530 & 0.503621 \\
\hline 18 & 5 & $(0,0,0,0,13)$ & 0.013741 & 0.027345 & 0.041801 & 0.057221 & 0.073743 \\
\hline 20 & 5 & $(15,0,0,0,0)$ & 0.012454 & 0.070280 & 0.155381 & 0.271032 & 0.502336 \\
\hline 20 & 5 & $(0,0,0,0,15)$ & 0.012454 & 0.024628 & 0.037478 & 0.051084 & 0.065541 \\
\hline
\end{tabular}


Table 3. Variances of progressively Type-II right censored order statistics.

\begin{tabular}{|c|c|c|c|c|c|c|c|}
\hline \multicolumn{8}{|c|}{$\beta=2$} \\
\hline$m \downarrow$ & $n \downarrow$ & Scheme & & & Variance & & \\
\hline 5 & 2 & $(0,3)$ & 0.011030 & 0.006372 & & & \\
\hline 5 & 2 & $(3,0)$ & 0.011030 & 0.054530 & & & \\
\hline 8 & 2 & $(6,0)$ & 0.000724 & 0.063226 & & & \\
\hline 8 & 2 & $(0,6)$ & 0.000724 & 0.002816 & & & \\
\hline 10 & 2 & $(8,0)$ & 0.000624 & 0.055025 & & & \\
\hline 10 & 2 & $(0,8)$ & 0.000624 & 0.001284 & & & \\
\hline 12 & 2 & $(10,0)$ & 0.000460 & 0.054761 & & & \\
\hline 12 & 2 & $(0,10)$ & 0.000460 & 0.000821 & & & \\
\hline 15 & 2 & $(13,0)$ & 0.000326 & 0.054628 & & & \\
\hline 15 & 2 & $(0,13)$ & 0.000326 & 0.000521 & & & \\
\hline 18 & 2 & $(16,0)$ & 0.000254 & 0.054554 & & & \\
\hline 18 & 2 & $(0,16)$ & 0.000254 & 0.000361 & & & \\
\hline 20 & 2 & $(18,0)$ & 0.000232 & 0.054524 & & & \\
\hline 20 & 2 & $(0,18)$ & 0.000232 & 0.000292 & & & \\
\hline 5 & 3 & $(2,0,0)$ & 0.003040 & 0.016404 & 0.070105 & & \\
\hline 5 & 3 & $(0,0,2)$ & 0.003040 & 0.005572 & 0.012217 & & \\
\hline 8 & 3 & $(5,0,0)$ & 0.000845 & 0.015101 & 0.068601 & & \\
\hline 8 & 3 & $(0,0,5)$ & 0.000845 & 0.002016 & 0.003502 & & \\
\hline 10 & 3 & $(7,0,0)$ & 0.000624 & 0.014801 & 0.068201 & & \\
\hline 10 & 3 & $(0,0,7)$ & 0.000624 & 0.001284 & 0.002120 & & \\
\hline 12 & 3 & $(9,0,0)$ & 0.000460 & 0.014635 & 0.068137 & & \\
\hline 12 & 3 & $(0,0,9)$ & 0.000460 & 0.000821 & 0.001437 & & \\
\hline 15 & 3 & $(12,0,0)$ & 0.000326 & 0.014502 & 0.068013 & & \\
\hline 15 & 3 & $(0,0,12)$ & 0.000326 & 0.000521 & 0.000836 & & \\
\hline 18 & 3 & $(15,0,0)$ & 0.000254 & 0.014531 & 0.068130 & & \\
\hline 18 & 3 & $(0,0,15)$ & 0.000254 & 0.000361 & 0.000562 & & \\
\hline 20 & 3 & $(17,0,0)$ & 0.000242 & 0.014418 & 0.068120 & & \\
\hline 20 & 3 & $(0,0,17)$ & 0.000242 & 0.000292 & 0.000436 & & \\
\hline 5 & 4 & $(1,0,0,0)$ & 0.003012 & 0.008173 & 0.022348 & 0.075850 & \\
\hline 5 & 4 & $(0,0,0,1)$ & 0.003031 & 0.005572 & 0.012317 & 0.025712 & \\
\hline 8 & 4 & $(4,0,0,0)$ & 0.000924 & 0.006960 & 0.022133 & 0.075435 & \\
\hline 8 & 4 & $(0,0,0,4)$ & 0.000924 & 0.002016 & 0.003502 & 0.005643 & \\
\hline 10 & 4 & $(6,0,0,0)$ & 0.000624 & 0.006368 & 0.020743 & 0.074245 & \\
\hline 10 & 4 & $(0,0,0,6)$ & 0.000624 & 0.001204 & 0.002120 & 0.003212 & \\
\hline 12 & 4 & $(8,0,0,0)$ & 0.000460 & 0.006514 & 0.021472 & 0.075170 & \\
\hline 12 & 4 & $(0,0,0,8)$ & 0.000460 & 0.000824 & 0.001437 & 0.002118 & \\
\hline 15 & 4 & $(11,0,0,0)$ & 0.000326 & 0.006271 & 0.020446 & 0.074148 & \\
\hline 15 & 4 & $(0,0,0,11)$ & 0.000326 & 0.000520 & 0.000836 & 0.001207 & \\
\hline 18 & 4 & $(14,0,0,0)$ & 0.000254 & 0.006218 & 0.020374 & 0.073875 & \\
\hline 18 & 4 & $(0,0,0,14)$ & 0.000254 & 0.000361 & 0.000561 & 0.000816 & \\
\hline 20 & 4 & $(16,0,0,0)$ & 0.000232 & 0.006167 & 0.020342 & 0.073843 & \\
\hline 20 & 4 & $(0,0,0,16)$ & 0.000232 & 0.000372 & 0.000456 & 0.000641 & \\
\hline 5 & 5 & $(0,0,0,0,0)$ & 0.003130 & 0.005572 & 0.012031 & 0.025712 & 0.079204 \\
\hline 8 & 5 & $(3,0,0,0,0)$ & 0.000924 & 0.004281 & 0.011013 & 0.024319 & 0.076112 \\
\hline 8 & 5 & $(0,0,0,0,3)$ & 0.000924 & 0.002016 & 0.003512 & 0.005643 & 0.008986 \\
\hline 10 & 5 & $(5,0,0,0,0)$ & 0.000624 & 0.004056 & 0.010801 & 0.025076 & 0.078511 \\
\hline 10 & 5 & $(0,0,0,0,5)$ & 0.000624 & 0.001284 & 0.002210 & 0.003212 & 0.004718 \\
\hline 12 & 5 & $(7,0,0,0,0)$ & 0.000860 & 0.003804 & 0.009748 & 0.024124 & 0.077425 \\
\hline 12 & 5 & $(0,0,0,0,7)$ & 0.000860 & 0.000822 & 0.001437 & 0.002108 & 0.002938 \\
\hline 15 & 5 & $(10,0,0,0,0)$ & 0.000326 & 0.003672 & 0.009615 & 0.023920 & 0.077311 \\
\hline 15 & 5 & $(0,0,0,0,10)$ & 0.000326 & 0.000521 & 0.000836 & 0.001217 & 0.001730 \\
\hline 18 & 5 & $(13,0,0,0,0)$ & 0.000254 & 0.003617 & 0.009542 & 0.023917 & 0.077221 \\
\hline 18 & 5 & $(0,0,0,0,13)$ & 0.000254 & 0.000361 & 0.000571 & 0.000806 & 0.001158 \\
\hline 20 & 5 & $(15,0,0,0,0)$ & 0.000234 & 0.003566 & 0.009511 & 0.023686 & 0.077187 \\
\hline 20 & 5 & $(0,0,0,0,15)$ & 0.000234 & 0.000290 & 0.000456 & 0.000641 & 0.000852 \\
\hline
\end{tabular}


Table 4. Variances of progressively Type-II right censored order statistics.

\begin{tabular}{|c|c|c|c|c|c|c|c|}
\hline \multicolumn{8}{|c|}{$\beta=4$} \\
\hline$m \downarrow$ & $n \downarrow$ & Scheme & & & Variance & & \\
\hline 5 & 2 & $(0,3)$ & 0.005102 & 0.011251 & & & \\
\hline 5 & 2 & $(3,0)$ & 0.005102 & 0.113001 & & & \\
\hline 8 & 2 & $(6,0)$ & 0.002453 & 0.110560 & & & \\
\hline 8 & 2 & $(0,6)$ & 0.002453 & 0.003716 & & & \\
\hline 10 & 2 & $(8,0)$ & 0.002101 & 0.110162 & & & \\
\hline 10 & 2 & $(0,8)$ & 0.002101 & 0.002325 & & & \\
\hline 12 & 2 & $(10,0)$ & 0.000784 & 0.110702 & & & \\
\hline 12 & 2 & $(0,10)$ & 0.000784 & 0.001611 & & & \\
\hline 15 & 2 & $(13,0)$ & 0.000533 & 0.110441 & & & \\
\hline 15 & 2 & $(0,13)$ & 0.000533 & 0.001144 & & & \\
\hline 18 & 2 & $(16,0)$ & 0.000407 & 0.110303 & & & \\
\hline 18 & 2 & $(0,16)$ & 0.000407 & 0.000664 & & & \\
\hline 20 & 2 & $(18,0)$ & 0.000340 & 0.110245 & & & \\
\hline 20 & 2 & $(0,18)$ & 0.000340 & 0.000536 & & & \\
\hline 5 & 3 & $(2,0,0)$ & 0.005103 & 0.030213 & 0.130125 & & \\
\hline 5 & 3 & $(0,0,2)$ & 0.005103 & 0.011150 & 0.022372 & & \\
\hline 8 & 3 & $(5,0,0)$ & 0.001652 & 0.027480 & 0.135586 & & \\
\hline 8 & 3 & $(0,0,5)$ & 0.001652 & 0.003716 & 0.006474 & & \\
\hline 10 & 3 & $(7,0,0)$ & 0.002103 & 0.027016 & 0.135022 & & \\
\hline 10 & 3 & $(0,0,7)$ & 0.002103 & 0.002325 & 0.003901 & & \\
\hline 12 & 3 & $(9,0,0)$ & 0.000784 & 0.026610 & 0.134616 & & \\
\hline 12 & 3 & $(0,0,9)$ & 0.000784 & 0.001611 & 0.002612 & & \\
\hline 15 & 3 & $(12,0,0)$ & 0.000533 & 0.026360 & 0.134466 & & \\
\hline 15 & 3 & $(0,0,12)$ & 0.000533 & 0.000964 & 0.001537 & & \\
\hline 18 & 3 & $(15,0,0)$ & 0.000407 & 0.026224 & 0.134330 & & \\
\hline 18 & 3 & $(0,0,15)$ & 0.000407 & 0.000664 & 0.001135 & & \\
\hline 20 & 3 & $(17,0,0)$ & 0.000340 & 0.026165 & 0.134271 & & \\
\hline 20 & 3 & $(0,0,17)$ & 0.000340 & 0.000536 & 0.000845 & & \\
\hline 5 & 4 & $(1,0,0,0)$ & 0.005113 & 0.016016 & 0.041042 & 0.151148 & \\
\hline 5 & 4 & $(0,0,0,1)$ & 0.005113 & 0.011151 & 0.022272 & 0.047320 & \\
\hline 8 & 4 & $(4,0,0,0)$ & 0.002453 & 0.013576 & 0.038602 & 0.146708 & \\
\hline 8 & 4 & $(0,0,0,4)$ & 0.002453 & 0.003706 & 0.006476 & 0.011281 & \\
\hline 10 & 4 & $(6,0,0,0)$ & 0.002110 & 0.013012 & 0.038041 & 0.146145 & \\
\hline 10 & 4 & $(0,0,0,6)$ & 0.002110 & 0.002325 & 0.003901 & 0.005933 & \\
\hline 12 & 4 & $(8,0,0,0)$ & 0.000784 & 0.012707 & 0.037733 & 0.145840 & \\
\hline 12 & 4 & $(0,0,0,8)$ & 0.000784 & 0.001611 & 0.002612 & 0.003848 & \\
\hline 15 & 4 & $(11,0,0,0)$ & 0.000533 & 0.013456 & 0.037483 & 0.145611 & \\
\hline 15 & 4 & $(0,0,0,11)$ & 0.000533 & 0.000964 & 0.001637 & 0.002342 & \\
\hline 18 & 4 & $(14,0,0,0)$ & 0.000417 & 0.012321 & 0.037347 & 0.145453 & \\
\hline 18 & 4 & $(0,0,0,14)$ & 0.000417 & 0.000664 & 0.001135 & 0.001581 & \\
\hline 20 & 4 & $(16,0,0,0)$ & 0.000341 & 0.012362 & 0.037288 & 0.145414 & \\
\hline 20 & 4 & $(0,0,0,16)$ & 0.000341 & 0.000536 & 0.000845 & 0.001271 & \\
\hline 5 & 5 & $(0,0,0,0,0)$ & 0.005113 & 0.011251 & 0.022372 & 0.047321 & 0.155405 \\
\hline 8 & 5 & $(3,0,0,0,0)$ & 0.002453 & 0.007911 & 0.019832 & 0.044860 & 0.153065 \\
\hline 8 & 5 & $(0,0,0,0,3)$ & 0.002453 & 0.003716 & 0.006476 & 0.011281 & 0.017537 \\
\hline 10 & 5 & $(5,0,0,0,0)$ & 0.002110 & 0.007435 & 0.021260 & 0.045205 & 0.161301 \\
\hline 10 & 5 & $(0,0,0,0,5)$ & 0.002110 & 0.002325 & 0.003911 & 0.005933 & 0.008733 \\
\hline 12 & 5 & $(7,0,0,0,0)$ & 0.000788 & 0.007041 & 0.019163 & 0.044121 & 0.152126 \\
\hline 12 & 5 & $(0,0,0,0,7)$ & 0.000788 & 0.001611 & 0.002612 & 0.003848 & 0.005412 \\
\hline 15 & 5 & $(10,0,0,0,0)$ & 0.000533 & 0.006812 & 0.018713 & 0.043740 & 0.151845 \\
\hline 15 & 5 & $(0,0,0,0,10)$ & 0.000533 & 0.000964 & 0.001637 & 0.002332 & 0.003161 \\
\hline 18 & 5 & $(13,0,0,0,0)$ & 0.000417 & 0.006654 & 0.018577 & 0.043613 & 0.151710 \\
\hline 18 & 5 & $(0,0,0,0,13)$ & 0.000417 & 0.000664 & 0.001135 & 0.001581 & 0.002111 \\
\hline 20 & 5 & $(15,0,0,0,0)$ & 0.000341 & 0.006641 & 0.018518 & 0.043545 & 0.151651 \\
\hline 20 & 5 & $(0,0,0,0,15)$ & 0.000341 & 0.000536 & 0.000845 & 0.001271 & 0.001662 \\
\hline
\end{tabular}




\section{Parameter Estimation under Progressive Type - II Censored Order Statistics}

5.1. Maximum Likelihood Method. Let $X_{1: m: n}, X_{2: m: n}, \ldots, X_{m: m: n}$ be a progressively Type-II censored sample from $R K D(\beta)$ with $\left(R_{1}, R_{2}, \ldots, R_{m}\right)$ being the progressive censoring scheme. The likelihood function based on the progressive censored sample is given by

$$
\begin{aligned}
& f_{X_{1: m: n}}, X_{2: m: n} \ldots X_{m: m: n}\left(x_{1}, x_{2}, \ldots, x_{m}\right) \\
& =A(n, m-1) \prod_{i=1}^{m} f\left(x_{i}\right)\left[1-F\left(x_{i}\right)\right]^{R_{i}} .
\end{aligned}
$$

where $f(x)$ and $F(x)$ are given respectively by eqns. (1.1) and (1.2). Substituting eqns. (1.1) and (1.2) into eqn. (5.1), the likelihood function is

$$
\begin{aligned}
L(\mathbf{x} \mid \beta) & =A(n, m-1) \prod_{i=1}^{m}\left\{\beta x_{i}^{\beta-1}\left(1-x_{i}\right)^{-\beta-1} e^{-\left(\frac{x_{i}}{1-x_{i}}\right)^{\beta}}\right\} \\
& \times\left[e^{-\left(\frac{x_{i}}{1-x_{i}}\right)^{\beta}}\right]^{R_{i}} .
\end{aligned}
$$

The log of likelihood function is

$$
\begin{aligned}
\ln L(\mathbf{x} \mid \beta) & =C+m \ln \beta+(\beta-1) \sum_{i=1}^{m} \ln \left(x_{i}\right)-(\beta+1) \sum_{i=1}^{m} \ln \left(1-x_{i}\right) \\
& -\sum_{i=1}^{m}\left(1+R_{i}\right)\left(\frac{x_{i}}{1-x_{i}}\right)^{\beta}
\end{aligned}
$$

where $C=\ln \{A(n, m-1)\}$. Upon differentiating (5.3) with respect to $\beta$ and equating to zero, the resulting equation must be satisfied to obtain MLE of $\beta$. The equation is given by

$$
\begin{aligned}
\frac{\partial \ln L(\mathbf{x} \mid \beta)}{\partial \beta} & =\frac{m}{\beta}+\sum_{i=1}^{m} \ln \left(x_{i}\right) \\
& -\sum_{i=1}^{m} \ln \left(1-x_{i}\right)-\sum_{i=1}^{m}\left(1+R_{i}\right)\left(\frac{x_{i}}{1-x_{i}}\right)^{\beta} \ln \left(\frac{x_{i}}{1-x_{i}}\right)=0 .
\end{aligned}
$$

Using large sample approximation, the asymptotic distribution of the $M L E$ is $[\sqrt{n}(\hat{\beta}-$ $\beta)] \rightarrow N\left(0, I^{-1}(\beta)\right)$, where $I^{-1}(\beta)$, is the inverse of the observed information matrix of the unknown parameter. The element of the observed information matrix is

$$
\frac{\partial^{2} \ln L(\mathbf{x} \mid \beta)}{\partial \beta^{2}}=\frac{-m}{\beta^{2}}-\sum_{i=1}^{m}\left(1+R_{i}\right)\left(\frac{x_{i}}{1-x_{i}}\right)^{\beta} \ln ^{2}\left(\frac{x_{i}}{1-x_{i}}\right) .
$$

The approximate $100(1-\tau) \%$ confidence intervals of the parameters $\beta$ is

$$
\hat{\beta} \pm z_{\tau / 2} \sqrt{\operatorname{var}(\hat{\beta})}
$$

where $\operatorname{var}(\hat{\beta})$ is obtained from $I^{-1}(\beta)$ and $z_{\tau / 2}$ is the upper $(\tau / 2)$ th percentile of the standard normal distribution.

5.2. Bayesian Estimation. This section discusses the Bayes procedure to derive the point and interval estimates of the parameter $\beta$ based on progressively Type-II censored data . In our Bayesian analysis, we have assumed three types of loss functions. In this article, the proposed prior for the parameters $\beta$ is considered as

$$
g(\beta) \propto \beta^{a-1} e^{-b \beta} ; \quad \beta, a, b>0 .
$$


The posterior distribution of $\beta$ is obtained after simplification as

$$
\pi(\beta \mid \mathbf{x})=\frac{1}{J_{1}} \beta^{m+a-1} e^{-\left[b \beta+\sum_{i=1}^{m}\left(1+R_{i}\right)\left(\frac{x_{i}}{1-x_{i}}\right)^{\beta}\right]} \prod_{i=1}^{m} x_{i}^{\beta-1}\left(1-x_{i}\right)^{-\beta-1} .
$$

where

$$
J_{1}=\int_{0}^{\infty} \beta^{m+a-1} e^{-\left[b \beta+\sum_{i=1}^{m}\left(1+R_{i}\right)\left(\frac{x_{i}}{1-x_{i}}\right)^{\beta}\right]} \prod_{i=1}^{m} x_{i}^{\beta-1}\left(1-x_{i}\right)^{-\beta-1} d \beta .
$$

We use three different loss functions to obtain the Bayes estimate of the unknown parameter $\beta$.

1- The first loss function is the symmetric squared error (SE) loss function. Using SE loss function, the Bayes estimate of the parameter $\beta$, denoted by $\tilde{\beta}_{S E}$, is the posterior mean.

2- The second loss function is the asymmetric LINEX loss function proposed by Varian (1975). Under LINEX loss function, the Bayes estimate of the parameter $\beta$, denoted by $\tilde{\beta}_{L I N E X}$ is given by

$$
\tilde{\beta}_{L I N E X}=-\frac{1}{v} \ln E\left(e^{-v \beta}\right),
$$

where $v \neq 0$ is constant.

3- The third loss function is the asymmetric general entropy (GE) loss function. The Bayes estimate of the parameter $\beta$, denoted by $\tilde{\beta}_{G E}$, in given by

$$
\tilde{\beta}_{G E}=\left[E\left(\beta^{-c}\right)\right]^{-1 / c},
$$

where $c$ is the shape parameter of the loss function, provided that $E\left(\beta^{-c}\right)$ exists.

From (5.8) and using the squared error loss function, the Bayes estimator of $\beta$ is given by

$$
\tilde{\beta}_{S E}=\frac{1}{J_{1}} \int_{0}^{\infty} \beta^{m+a} e^{-\left[b \beta+\sum_{i=1}^{m}\left(1+R_{i}\right)\left(\frac{x_{i}}{1-x_{i}}\right)^{\beta}\right]} \prod_{i=1}^{m} x_{i}^{\beta-1}\left(1-x_{i}\right)^{-\beta-1} d \beta .
$$

Similarlyy, from (5.8) and (5.10), the Bayes estimator of $\beta$ under LINEX loss function is given by

$$
\begin{aligned}
\tilde{\beta}_{L I N E X} & =-\frac{1}{v} \ln \left[\frac{1}{J_{1}} \int_{0}^{\infty} \beta^{m+a-1} e^{-\left[v \beta+b \beta+\sum_{i=1}^{m}\left(1+R_{i}\right)\left(\frac{x_{i}}{1-x_{i}}\right)^{\beta}\right]}\right. \\
& \left.\times \prod_{i=1}^{m} x_{i}^{\beta-1}\left(1-x_{i}\right)^{-\beta-1} d \beta\right] .
\end{aligned}
$$

Using (5.8) and (5.11), the Bayes estimator of $\beta$ under GE loss function is given by

$$
\begin{aligned}
\tilde{\beta}_{G E} & =\left[\frac{1}{J_{1}} \int_{0}^{\infty} \beta^{m+a-c-1} e^{-\left[b \beta+\sum_{i=1}^{m}\left(1+R_{i}\right)\left(\frac{x_{i}}{1-x_{i}}\right)^{\beta}\right]}\right. \\
& \left.\times \prod_{i=1}^{m} x_{i}^{\beta-1}\left(1-x_{i}\right)^{-\beta-1} d \beta\right]^{-1 / c} .
\end{aligned}
$$

The Bayesian method of interval estimation is more straightforward than the classical method of confidence intervals. Once the posterior distribution of $\beta$ has been obtained, a symmetric $100(1-\tau) \%$ two-sided Bayes probability interval (TBPI) of $\beta$, denoted by $\left[\beta_{L}, \beta_{U}\right]$, can be obtained by solving the following two equations (see [27], page 208-209).

$$
\int_{0}^{\beta_{L}} \pi(\theta \mid x) d \beta=\frac{\tau}{2}, \int_{\beta_{U}}^{\infty} \pi(\beta \mid x) d \beta=\frac{\tau}{2}
$$


for the limits $\beta_{L}$ and $\beta_{U}$. Now we compute the highest posterior density (HPD) credible intervals for $\beta$. Since $\pi(\beta \mid x)$ is unimodal, the corresponding $100(1-\gamma) \%$ HPD credible interval $\left[H_{L}^{\beta}, H_{U}^{\beta}\right]$ can be obtained from the simultaneous solution of the following equations

$$
P\left(H_{L}^{\beta}<\beta<H_{U}^{\beta}\right]=\int_{H_{L}^{\beta}}^{H_{U}^{\beta}} \pi(\beta \mid x) d \beta=1-\gamma,
$$

and

$$
\pi\left(H_{L}^{\beta} \mid x\right)=\pi\left(H_{U}^{\beta} \mid x\right)
$$

\section{Simulation Study}

In this section, a simulation study is conducted to study the behaviour of the ML and Bayes estimates under the different loss function by considering $(n, m)=(30,5),(30,10)$, $(45,5),(45,15),(60,10)$ and $(60,20)$ and different values of the parameter $\beta$, where $\beta=$ $1.5,3$ in all the cases. We have obtained the ML and Bayes estimates by using the following progressive censoring schemes

- Scheme 1: $R_{1}=\cdots=R_{m}=\frac{n-m}{m}$.

- Scheme 2: $R_{1}=\cdots=R_{m-1}=1$ and $R_{m}=n-2 m+1$.

- Scheme 3: $R_{1}=\cdots=R_{m-1}=0$ and $R_{m}=n-m$.

We use the algorithm introduced by [4] to generate progressively censored reduced Kies samples. We consider three types of priors to obtain the Bayes estimates, Prior 0: $a=$ $b=c=d=0$, which describes the case of non-informative prior. We use Prior 0 to obtain the Bayes estimates for the two values of the parameter $\beta$. Prior $1: a=3, b=2$ to obtain the Bayes estimates for $\beta=1.5$ and Prior $2: a=1.5, b=0.5$ to obtain the Bayes estimates when $\beta=3$. It is to be noted that, prior 1 and prior 2 describe the case of informative prior. In each setting, we obtain the MLEs and Bayes estimates under SE, LINEX $(v=0.5)$ and GE $(c=0.5)$ loss functions. The process is replicated 1000 times. The average values of the estimates, mean squared errors (MSEs), confidence/ credible interval lengths and coverage probabilities are obtained and tabulated.

The average values of the estimates and the corresponding MSEs are displayed in Table 5 for $\beta=1.5$ and in Table 7 for $\beta=3$. The average confidence/ credible interval lengths and the corresponding coverage probabilities are presented in Table 6 for $\beta=1.5$ and in Table 8 for $\beta=3$. From Tables $5-8$, it is to be noted that the Bayes estimates under SE loss function under Prior 0 is quite close to the MLEs. In terms of MSEs and confidence/credible interval lengths, the Bayes estimates using the informative priors (i.e. Prior 1 and Prior 2) perform better than those based on the non-informative prior (Prior 0 ) and the MLEs for two parameter values of $\beta$. The Bayes estimates under LINEX loss function have the smallest MSEs for all cases when $\beta=3$, and in some cases when $\beta=1.5$. For fixed $n$, when the number of observed failure $m$ increases, the MSEs and the confidence/credible interval lengths decreases in all cases. Comparing the three censoring schemes, it is clear that the MSEs, confidence/credible interval lengths are smaller for Scheme 1 than Schemes 2 and 3.

\section{Real Data Analysis}

In this section we analyze a real data set given by [31] and also studied by [23]. The original data consists of 40 observations and it describes the strength of a kind of glass, which were measured by three-point flexural method. From the complete data set, we generate three progressively censored samples from $n=40$ and $m=10$ according to the 
Table 5. Average values of different estimators and the corresponding MSEs (in parentheses) for $\beta=1.5$.

\begin{tabular}{|c|c|c|c|c|c|c|c|c|}
\hline \multirow[b]{2}{*}{$(n, m)$} & \multirow[b]{2}{*}{ Scheme } & \multirow[t]{2}{*}{ ML Estimate } & \multicolumn{3}{|c|}{ Bayes Estimates (Prior 0) } & \multicolumn{3}{|c|}{ Bayes Estimates (Prior 1) } \\
\hline & & & $\mathrm{SE}$ & LINEX & GE & $\mathrm{SE}$ & LINEX & GE \\
\hline \multirow{3}{*}{$(30,5)$} & 1 & $1.565(0.134)$ & $1.561(0.132)$ & $1.529(0.119)$ & $1.502(0.119)$ & $1.543(0.093)$ & $1.517(0.085)$ & $1.507(0.085)$ \\
\hline & 2 & $1.565(0.136)$ & $1.562(0.135)$ & $1.529(0.121)$ & $1.501(0.121)$ & $1.544(0.094)$ & $1.517(0.086)$ & $1.508(0.086)$ \\
\hline & 3 & $1.559(0.147)$ & $1.567(0.149)$ & $1.531(0.133)$ & $1.500(0.133)$ & $1.545(0.099)$ & $1.515(0.091)$ & $1.512(0.091)$ \\
\hline \multirow[t]{3}{*}{$(30,10)$} & 1 & $1.591(0.139)$ & $1.579(0.135)$ & $1.548(0.120)$ & $1.521(0.119)$ & $1.560(0.095)$ & $1.534(0.086)$ & $1.511(0.085)$ \\
\hline & 2 & $1.586(0.137)$ & $1.579(0.135)$ & $1.548(0.121)$ & $1.520(0.119)$ & $1.559(0.095)$ & $1.534(0.086)$ & $1.509(0.086)$ \\
\hline & 3 & $1.579(0.132)$ & $1.576(0.131)$ & $1.546(0.117)$ & $1.519(0.117)$ & $1.558(0.093)$ & $1.532(0.084)$ & $1.509(0.084)$ \\
\hline \multirow[t]{3}{*}{$(45,5)$} & 1 & $1.539(0.091)$ & $1.545(0.093)$ & $1.524(0.086)$ & $1.503(0.086)$ & $1.535(0.072)$ & $1.515(0.068)$ & $1.503(0.068)$ \\
\hline & 2 & $1.539(0.093)$ & $1.546(0.094)$ & $1.524(0.087)$ & $1.503(0.087)$ & $1.536(0.073)$ & $1.515(0.069)$ & $1.504(0.069)$ \\
\hline & 3 & $1.539(0.106)$ & $1.552(0.108)$ & $1.526(0.099)$ & $1.504(0.099)$ & $1.538(0.081)$ & $1.516(0.075)$ & $1.504(0.075)$ \\
\hline \multirow{3}{*}{$(45,15)$} & 1 & $1.561(0.099)$ & $1.553(0.098)$ & $1.533(0.091)$ & $1.515(0.091)$ & $1.543(0.077)$ & $1.526(0.072)$ & $1.511(0.072)$ \\
\hline & 2 & $1.557(0.100)$ & $1.553(0.099)$ & $1.532(0.092)$ & $1.514(0.092)$ & $1.544(0.078)$ & $1.525(0.073)$ & $1.508(0.072)$ \\
\hline & 3 & $1.549(0.092)$ & $1.547(0.092)$ & $1.528(0.085)$ & $1.511(0.085)$ & $1.538(0.073)$ & $1.520(0.069)$ & $1.505(0.070)$ \\
\hline \multirow[t]{3}{*}{$(60,10)$} & 1 & $1.555(0.071)$ & $1.552(0.071)$ & $1.539(0.068)$ & $1.528(0.068)$ & $1.546(0.059)$ & $1.532(0.057)$ & $1.521(0.058)$ \\
\hline & 2 & $1.556(0.073)$ & $1.554(0.073)$ & $1.539(0.068)$ & $1.524(0.067)$ & $1.547(0.062)$ & $1.533(0.059)$ & $1.521(0.061)$ \\
\hline & 3 & $1.557(0.081)$ & $1.560(0.082)$ & $1.543(0.076)$ & $1.527(0.075)$ & $1.552(0.067)$ & $1.535(0.063)$ & $1.522(0.067)$ \\
\hline \multirow[t]{3}{*}{$(60,20)$} & 1 & $1.541(0.059)$ & $1.534(0.059)$ & $1.521(0.055)$ & $1.507(0.055)$ & $1.529(0.050)$ & $1.515(0.048)$ & $1.504(0.047)$ \\
\hline & 2 & $1.539(0.061)$ & $1.536(0.061)$ & $1.522(0.059)$ & $1.509(0.059)$ & $1.531(0.053)$ & $1.517(0.049)$ & $1.505(0.051)$ \\
\hline & 3 & $1.539(0.062)$ & $1.538(0.061)$ & $1.521(0.059)$ & $1.510(0.061)$ & $1.533(0.053)$ & $1.519(0.051)$ & $1.508(0.053)$ \\
\hline
\end{tabular}

Table 6. Average confidence interval/credible interval lengths and the coverage percentages (in parentheses) for $\beta=1.5$.

\begin{tabular}{ccccccc}
\hline$(n, m)$ & Scheme & Approximate & Symmetric Credible Interval & \multicolumn{2}{c}{ HPD Interval } \\
\hline \multirow{2}{*}{$(30,5)$} & 1 & $1.3212(93.70)$ & $1.3468(95.30)$ & $1.2455(97.20)$ & $1.3803(94.90)$ & $1.2653(96.80)$ \\
& 2 & $1.3362(93.60)$ & $1.3586(95.10)$ & $1.2586(97.10)$ & $1.3929(95.00)$ & $1.2772(96.60)$ \\
& 3 & $1.4082(93.30)$ & $1.4359(94.90)$ & $1.3167(97.10)$ & $1.4653(95.30)$ & $1.3296(96.50)$ \\
$(30,10)$ & 1 & $1.2860(93.00)$ & $1.3187(94.40)$ & $1.2089(97.00)$ & $1.3231(94.90)$ & $1.1930(95.70)$ \\
& 2 & $1.3016(93.00)$ & $1.3321(95.00)$ & $1.2277(96.60)$ & $1.3391(94.70)$ & $1.2270(96.20)$ \\
& 3 & $1.2846(93.40)$ & $1.3092(94.70)$ & $1.2154(96.10)$ & $1.3431(94.80)$ & $1.2312(96.10)$ \\
$(45,5)$ & 1 & $1.1211(94.40)$ & $1.0581(95.00)$ & $1.0208(95.90)$ & $1.0857(92.70)$ & $1.0848(95.40)$ \\
& 2 & $1.1291(94.40)$ & $1.0694(94.90)$ & $1.0245(95.90)$ & $1.1609(94.30)$ & $1.0920(95.40)$ \\
$(45,15)$ & 3 & $1.2092(94.50)$ & $1.1273(94.70)$ & $1.0667(96.00)$ & $1.2417(94.20)$ & $1.1566(95.70)$ \\
& 1 & $1.0509(92.70)$ & $0.8137(94.00)$ & $0.9453(95.20)$ & $1.0652(96.40)$ & $1.0129(97.00)$ \\
$(60,10)$ & 2 & $1.0660(92.50)$ & $1.0433(94.40)$ & $0.9671(94.80)$ & $1.0799(96.20)$ & $1.0266(96.80)$ \\
& 1 & $1.0496(92.90)$ & $1.0269(94.50)$ & $0.9640(95.30)$ & $1.0881(95.90)$ & $1.0351(96.90)$ \\
$(60,20)$ & 2 & $0.9263(93.50)$ & $0.8173(94.80)$ & $0.8526(95.10)$ & $0.9388(94.00)$ & $0.9022(94.30)$ \\
& 3 & $0.9917(93.30)$ & $0.9261(94.80)$ & $0.8967(95.40)$ & $0.9507(94.20)$ & $0.9132(94.30)$ \\
& 1 & $0.9069(94.20)$ & $1.0175(94.90)$ & $0.9486(95.80)$ & $1.0083(93.90)$ & $0.9630(94.40)$ \\
& 2 & $0.9211(93.90)$ & $0.88813(95.60)$ & $0.8707(96.60)$ & $0.9287(94.00)$ & $0.8974(95.30)$ \\
& 3 & $0.9083(93.20)$ & $0.8473(95.00)$ & $0.8108(95.80)$ & $0.9590(93.50)$ & $0.9151(95.30)$ \\
\hline
\end{tabular}

three censoring schemes discussed in section (6). The generated progressively censored samples are given in the following table

\begin{tabular}{lc}
\hline Scheme & Censored data \\
\hline 1 & $0.477,0.502,0.524,0.525,0.529,0.538,0.546,0.555,0.611,0.624$ \\
2 & $0.477,0.502,0.524,0.525,0.529,0.538,0.539,0.546,0.575,0.600$ \\
3 & $0.477,0.502,0.524,0.525,0.529,0.538,0.539,0.546,0.547,0.549$ \\
\hline
\end{tabular}

The MLE and Bayes estimates and the corresponding confidence/ credible intervals are reported in Tables 9 and 10, respectively. The Bayes estimates under SE, LINEX and GE loss functions are obtained based on a non-informative prior, because we have no information about the unknown parameter $\beta$. Figure 1 display the posterior distribution 
Table 7. Average values of different estimators and the corresponding MSEs (in parentheses) for $\beta=3$.

\begin{tabular}{|c|c|c|c|c|c|c|c|c|}
\hline \multirow[b]{2}{*}{$(n, m)$} & \multirow[b]{2}{*}{ Scheme } & \multirow[t]{2}{*}{ ML Estimate } & \multicolumn{3}{|c|}{ Bayes Estimates (Prior 0) } & \multicolumn{3}{|c|}{ Bayes Estimates (Prior 2) } \\
\hline & & & $\mathrm{SE}$ & LINEX & GE & $\mathrm{SE}$ & LINEX & GE \\
\hline \multirow[t]{3}{*}{$(30,5)$} & 1 & $3.166(0.558)$ & $3.159(0.555)$ & $3.033(0.449)$ & $3.039(0.492)$ & $3.137(0.458)$ & $3.023(0.378)$ & $3.026(0.409)$ \\
\hline & 2 & $3.167(0.573)$ & $3.163(0.571)$ & $3.034(0.461)$ & $039(0.505)$ & $3.139(0.469)$ & $3.022(0.386)$ & 419) \\
\hline & 3 & $3.168(0.647)$ & $3.183(0.657)$ & $3.039(0.517)$ & $3.047(0.575)$ & $3.153(0.525)$ & $3.024(0.424)$ & $3.029(0.465)$ \\
\hline \multirow{3}{*}{$(30,10)$} & 1 & $3.142(0.529)$ & $3.117(0.514)$ & $3.000(0.421)$ & $3.004(0.463)$ & $3.100(0.423)$ & $2.992(0.353)$ & $2.996(0.383)$ \\
\hline & 2 & $3.134(0.523)$ & $3.120(0.515)$ & $3.000(0.421)$ & $3.004(0.463)$ & $3.102(0.423)$ & $2.992(0.352)$ & $2.994(0.383)$ \\
\hline & 3 & $3.119(0.465)$ & $3.113(0.460)$ & $2.997(0.383)$ & $3.001(0.416)$ & $3.096(0.387)$ & $2.989(0.327)$ & $2.992(0.353)$ \\
\hline \multirow[t]{3}{*}{$(45,5)$} & 1 & $3.095(0.399)$ & $3.108(0.406)$ & $3.019(0.351)$ & $3.025(0.377)$ & $3.097(0.356)$ & $3.012(0.309)$ & $3.014(0.334)$ \\
\hline & 2 & $3.096(0.405)$ & $3.109(0.411)$ & $3.021(0.355)$ & $3.023(0.381)$ & $3.097(0.361)$ & $3.013(0.314)$ & $3.014(0.336)$ \\
\hline & 3 & $3.102(0.465)$ & $3.127(0.478)$ & $3.025(0.403)$ & $3.029(0.435)$ & $3.109(0.408)$ & $3.015(0.349)$ & $3.020(0.375)$ \\
\hline \multirow[t]{3}{*}{$(45,15)$} & 1 & $3.091(0.316)$ & $3.075(0.311)$ & $2.999(0.275)$ & $2.999(0.294)$ & $3.068(0.277)$ & $2.996(0.247)$ & $2.995(0.263)$ \\
\hline & 2 & $3.084(0.319)$ & $3.074(0.316)$ & $2.997(0.279)$ & $2.999(0.295)$ & $3.068(0.282)$ & $2.992(0.251)$ & $2.995(0.268)$ \\
\hline & 3 & $3.074(0.302)$ & $3.070(0.301)$ & $2.995(0.267)$ & $2.996(0.282)$ & $3.025(0.397)$ & $2.991(0.241)$ & $2.993(0.254)$ \\
\hline \multirow{3}{*}{$(60,10)$} & 1 & $3.111(0.289)$ & $3.108(0.299)$ & $3.049(0.265)$ & $3.048(0.294)$ & $3.102(0.273)$ & $3.044(0.244)$ & $3.044(0.274)$ \\
\hline & 2 & $3.113(0.297)$ & $3.109(0.299)$ & $3.051(0.267)$ & $3.065(0.418)$ & $3.104(0.272)$ & $3.045(0.246)$ & $3.051(0.276)$ \\
\hline & 3 & $3.113(0.326)$ & $3.122(0.330)$ & $3.050(0.290)$ & $3.054(0.313)$ & $3.111(0.298)$ & $3.047(0.265)$ & $3.052(0.288)$ \\
\hline \multirow[t]{3}{*}{$(60,20)$} & 1 & $3.089(0.236)$ & $3.075(0.236)$ & $3.022(0.213)$ & $3.024(0.232)$ & $3.068(0.223)$ & $3.018(0.198)$ & $3.021(0.221)$ \\
\hline & 2 & $3.086(0.244)$ & $3.081(0.246)$ & $3.020(0.220)$ & $3.024(0.249)$ & $3.077(0.226)$ & $3.017(0.205)$ & $3.022(0.236)$ \\
\hline & 3 & $3.080(0.242)$ & $3.078(0.243)$ & $3.021(0.218)$ & $3.021(0.229)$ & $3.072(0.222)$ & $3.018(0.202)$ & $3.021(0.213)$ \\
\hline
\end{tabular}

Table 8. Average confidence interval/credible interval lengths and the coverage percentages (in parentheses) for $\beta=3$.

\begin{tabular}{ccccccc}
\hline$(n, m)$ & Scheme & Approximate & Symmetric Credible Interval & \multicolumn{2}{c}{ HPD Interval } \\
\hline \multirow{2}{*}{$(30,5)$} & 1 & $2.6396(93.00)$ & $3.1169(95.30)$ & $2.4761(97.00)$ & $3.1710(95.10)$ & $2.9930(95.80)$ \\
& 2 & $2.6696(93.00)$ & $3.0261(95.10)$ & $2.4920(96.60)$ & $3.1894(95.30)$ & $3.0440(95.80)$ \\
& 3 & $2.8161(92.70)$ & $3.4086(94.60)$ & $2.7255(96.00)$ & $3.3785(95.90)$ & $3.0588(95.90)$ \\
$(30,10)$ & 1 & $2.5681(94.00)$ & $3.0935(95.90)$ & $2.2692(96.80)$ & $2.7698(95.90)$ & $2.5529(96.20)$ \\
& 2 & $2.6016(94.00)$ & $3.1084(96.30)$ & $2.3806(97.00)$ & $2.7716(95.90)$ & $2.6959(96.10)$ \\
& 3 & $2.5697(93.90)$ & $2.9045(96.20)$ & $2.5833(97.10)$ & $2.7845(95.60)$ & $2.7456(96.00)$ \\
$(45,5)$ & 1 & $2.2436(93.80)$ & $2.6012(94.20)$ & $1.5559(94.50)$ & $2.2890(96.10)$ & $2.2578(96.80)$ \\
& 2 & $2.2591(93.60)$ & $2.6268(94.20)$ & $1.6068(94.60)$ & $2.3033(96.10)$ & $2.3044(96.70)$ \\
$(45,15)$ & 3 & $2.4147(93.50)$ & $2.9079(94.60)$ & $2.0136(95.20)$ & $2.4747(96.60)$ & $2.3717(96.70)$ \\
& 1 & $2.0987(94.90)$ & $2.2901(96.20)$ & $1.6122(95.90)$ & $2.1663(95.20)$ & $2.0994(95.90)$ \\
$(60,10)$ & 3 & $2.1296(94.50)$ & $2.3078(96.20)$ & $1.6767(95.60)$ & $2.5561(95.80)$ & $2.1478(96.50)$ \\
& 1 & $1.8536(93.40)$ & $2.2467(96.60)$ & $1.7197(96.30)$ & $2.6493(96.50)$ & $2.2090(96.90)$ \\
& 2 & $1.8766(93.30)$ & $2.0157(94.10)$ & $1.3082(93.10)$ & $2.0682(94.70)$ & $1.8823(95.30)$ \\
$(60,20)$ & 3 & $1.9843(93.20)$ & $2.2553(94.90)$ & $1.1414(93.00)$ & $2.0808(95.00)$ & $2.0128(95.40)$ \\
& 1 & $1.8142(93.80)$ & $1.9073(95.50)$ & $1.3480(93.70)$ & $2.1085(95.00)$ & $2.0843(95.40)$ \\
& 2 & $1.8419(93.80)$ & $1.9539(95.10)$ & $1.4656(93.20)$ & $1.8319(95.90)$ & $1.7980(96.10)$ \\
& 3 & $1.8146(93.60)$ & $1.9355(95.00)$ & $2.9850(94.10)$ & $1.8590(96.20)$ & $1.8238(96.30)$ \\
& & & & & & \\
& & & & & &
\end{tabular}

of $\beta$ for different censoring schemes. From Table 10 and Figure 1, it is observed that the symmetric and HDP intervals are coincide because the posterior distribution of $\beta$ is approximately symmetric.

Table 9. MLE and Bayes Estimates and the corresponding interval/credible intervals (in parentheses) of $\beta$.

\begin{tabular}{lcccc}
\hline Scheme & MLE & Bayes (SE) & Bayes (LINEX) & Bayes (GE) \\
\hline Complete & 1.3447 & 1.3326 & 1.3283 & 1.3227 \\
1 & 1.1403 & 1.1117 & 1.0904 & 1.0513 \\
2 & 0.7621 & 0.7617 & 0.7519 & 0.7154 \\
3 & 1.2731 & 1.2501 & 1.2205 & 1.1748 \\
\hline
\end{tabular}


Table 10. The approximate confidence /symmetric and HPD intervals of $\beta$.

\begin{tabular}{lccc}
\hline Scheme & MLE & Symmetric Credible Interval & HPD Interval \\
\hline Complete & $(1.1055,1.5839)$ & $(1.0763,1.5909)$ & $(1.0756,1.5901)$ \\
1 & $(0.5137,1.7669)$ & $(0.5968,1.7097)$ & $(0.5600,1.6898)$ \\
2 & $(0.2714,1.2528)$ & $(0.3875,1.1860)$ & $(0.4073,1.0785)$ \\
3 & $(0.6987,1.8475)$ & $(0.6439,1.9976)$ & $(0.6022,1.9383)$ \\
\hline
\end{tabular}

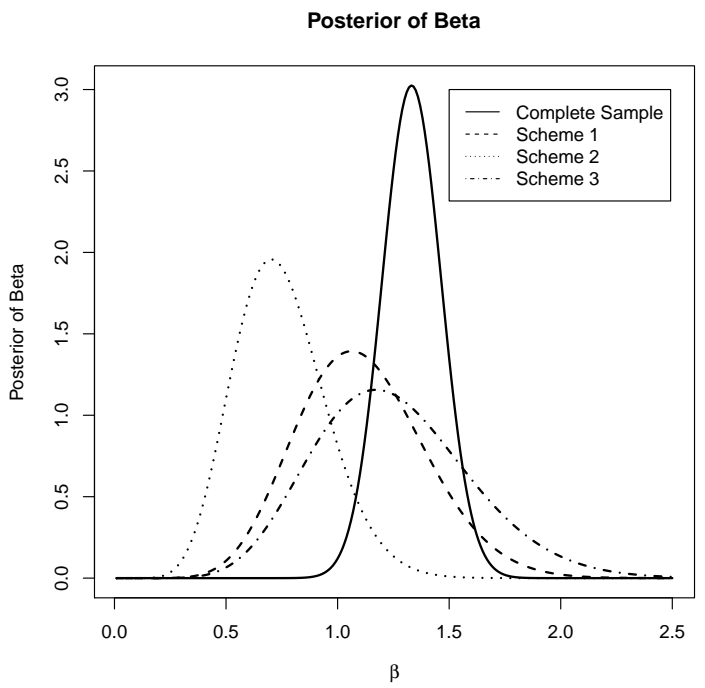

Figure 1. Posterior distribution of $\beta$ for different censoring schemes of [31] data.

Figure 1 shows the effect of censoring schemes on the shape of the posterior distribution of $\beta$. It can be seen that the shape of posterior distribution of $\beta$ based on scheme 2 shows a shift towards the right more than that of complete sample and other censoring schemes. Also, it can be is observed from Table 9 that the estimate of $\beta$ using scheme 2 is the lowest when compared with the complete sample and the other censoring schemes.

\section{Concluding Remarks.}

In this paper, we have provided explicit expressions and recurrence relations for single and product moments of progressively type-II censored samples of the reduced Kies distribution. We also characterized the distribution by means of recurrence relation. In addition, estimation of unknown parameter of the Reduced Kies distribution has been considered. We have compared the MLEs and different Bayes estimators with respect to the mean squared errors. We have also compared the asymptotic confidence intervals with the two-sided Bayes probability intervals and HPDI obtained from the posterior distribution functions. The simulation study shows that Bayes estimates performs better than the MLEs with regard to mean squared errors. The two-sided Bayes probability 
intervals are also of shorter length with competitive coverage percentages of the true parameter than the confidence intervals. Using a real data set, we demonstrated that proposed Bayes estimators perform better than MLE.

\section{Acknowledgements}

The authors would like to thank the editor and reviwers for their valuable and very constructive comments, which have greatly improved the contents of the paper.

\section{References}

[1] Aggarwala, R. and Balakrishnan, N. Recurrence relations for single and product moments of progressively Type-II censored order statistics from a exponential and truncated exponential distribution, Ann. Inst. Statist. Math., 48, 757-771, (1996).

[2] Arnold, B. C., Balakrishnan, N. and Nagaraja, H. N. A First Course in Order Statistics, John Wiley \& Sons, New York, (1992).

[3] Balakrishnan, N. and Malik, H. J. Order statistics from the linear-exponential distribution, part I: Increasing hazard rate case, Communications in Statistics, Theory and Methods, 15, 179-203, (1986).

[4] Balakrishnan, N. and Sandhu, R. A. A simple simulational algorithm for generating progressive Type-II censored samples, Amer. Statist., 49, 229-230, (1995).

[5] Balakrishnan, N. and Aggarwala, R. Recurrence relations for single and product moments of order statistics from a generalized logistic distribution with applications to inference and generalizations to double truncation, Handbook of Statistics, 17, 85-126., (1998).

[6] Balakrishnan, N. and Sultan, K. S. Recurrence relations for single and product moments of progressively Type-II censored order statistics from a exponential and truncated exponential distribution, Ann. Inst. Statist. Math., 48, 757-771, (1998).

[7] Balakrishnan, N. and Sultan, K. S. Recurrence relations and identities for moments of order statistics, In: N. Balakrishnan and C. R. Rao, eds. Handbook of Statistics, 16, 149 228, Order Statistics: Theory and Methods, North-Holland, Amsterdam, The Netherlands., (1998).

[8] Balakrishnan, N. and Aggarwala, R. Progressive Censoring: Theory, Method and Applications Birkhauser, Boston., (2000).

[9] Balakrishnan N, Lin C. T. On the distribution of a test for exponentiality based on progressively type-II right censored spacings, J. Stat. Comput. Simul. 73, 277-283, (2003).

[10] Balakrishnan, N., Ng, H. K.T. and Kannan, N. A test of exponentiality based on spacings for progressively type-II censored data. In: Huber-Carol C et al (eds) Goodness-of-fit tests and model validity. Birkhauser, Boston, (2002).

[11] Balakrishnan, N., Kannan, N., Lin, C. T., and Wu, S. J. S. Inference for the extreme value distribution under progressive type-II censoring, Journal of Statistical Computation \& Simulation, 74, 25-45, (2004).

[12] Balakrishnan, N. Progressive Censoring Methodology: An Appraisal Test, 16, 211-296 (with discussion), (2007).

[13] Balakrishnan, N., AL-Hussaini, E. K. and Saleh, H. M. Recurrence relations for moments of progressively censored order statistics from logistic distribution with applications to inference, Journal of Statistical Planning and Inference, 141(1), 17-30, (2011).

[14] Balakrishnan, N. and Saleh, H. M. Recurrence relations for single and product moments of progressively Type-II censored order statistics from a generalized halflogistic distribution with application to inference, Journal of Statistical Computation and Simulation , 83, 17041721, (2013).

[15] Cohen, A.C. Progressively censored samples in life testing, Technometrics, 5, 327-329, (1963).

[16] David, H. A. and Nagaraja, H. N. Order Statistics, Third Edition, John Wiley \& Sons, New York. 119, 171-189, (2003).

[17] Fernandez, A. J. On estimating exponential parameters with general type II progressive censoring, Journal of Statistical Planning and Inference, 121, 135-147, (2004). 
[18] Joshi, P. C. Recurrence relations between moments of order statistics from exponential and truncated exponential distributions, Sankhya Set. B, 39, 362-371, (1978).

[19] Kies, J.A. The strength of glass performance. Naval Research Lab Report No. 5093, Washington, D.C., (1958).

[20] Kumar, D., Dey, S., and Nadarajah, S. Extended exponential distribution based on order statistics, Communications in Statistics- Theory and Methods, 46, 9166-9184, (2017).

[21] Kumar, C. S and Dharmaja, S. H. S. On reduced Kies distribution. Collection of Recent Statistical Methods and Applications, (C.S. Kumar, M. Chacko and E.I.A. Sathar, Eds.), 111-123, Department of Statistics, University of Kerala Publishers, Trivandrum. (2013).

[22] Kumar, C. S and Dharmaja, S. H. S. On some properties of Kies distribution, Metron, 72, 97-122, (2014).

[23] Kumar, C. S and Dharmaja, S. H. S. The Exponentiated Reduced Kies distribution- Properties and Applications, Communications in Statistics-Theory and Methods, 46(17), 87788790, (2016).

[24] Mahmoud, R. M., Sultan, K. S. and Saleh, H. M. Progressively censored data from the linear exponential distribution, moments and estimation, Metorn, LXIV(2), 199-215, (2006).

[25] Malik, M.R and Kumar, D. Relations for moments of progressively Type-II right censored order statistics from Erlang-truncated exponential distribution, Statistics in Transition New Series, 18, 651-668, (2017).

[26] Mann, N. R. Best linear invariant estimation for Weibull parameters under progressive censoring, Technometrics, 13, 521-534, (1971).

[27] Martz, H., and Waller, R. Bayesian Reliability Analysis, New York: John Wiley, (1982).

[28] Sultan, K. S., Mahmoud, M. R. and Saleh, H. M. Moments of estimation from progressively censored data of the half logistic distribution, International Journal of Reliability and Applications, 7(2), 187-201, (2006).

[29] Thomas, D. R. and Wilson, W. M. Linear order statistic estimation for the two-parameter Weibull and extreme-value distributions from Type-II progressively censored samples, Technometrics, 14, 679-691, (1972).

[30] Viveros, R. and Balakrishnan, N. Interval estimation of life characteristics from progressively censored data, Technometrics, 36, 84-91, (1994).

[31] Xu, Y., Cheng, L., Zhang, L., Yan, D. and You, C. Optimization of sample number for Weibull functions of brittle materials strength, Ceramics International, 27, 239-241, (2001). 